\title{
Adherence to a Mediterranean Diet is Associated with a Lower Risk of Later-onset Crohn's Disease: Results From Two Large Prospective Cohort Studies
}

Hamed Khalili1'-4, Niclas Hakansson ${ }^{4}$, Simon S Chan ${ }^{5,6}$, Ye Chen ${ }^{2}$, Paul Lochhead ${ }^{1-2}$, Jonas F Ludvigsson $^{7-8}$, Andrew T Chan ${ }^{1-2}$, Andrew R Hart ${ }^{5,6}$, Ola Olen ${ }^{3,9}$, Alicja Wolk ${ }^{4,10}$

${ }^{1}$ Gastroenterology Unit, Massachusetts General Hospital, Harvard Medical School, Boston, Massachusetts, USA

${ }^{2}$ Clinical and Translational Epidemiology Unit, Massachusetts General Hospital, Harvard Medical School, Boston, Massachusetts, USA

${ }^{3}$ Clinical Epidemiology Division, Department of Medicine Solna, Karolinska Institutet, Stockholm, Sweden

${ }^{4}$ Institute of Environmental Medicine, Karolinska Institutet, Stockholm, Sweden

${ }^{5}$ Department of Medicine, Norwich Medical School, University of East Anglia, Norwich, United Kingdom

${ }^{6}$ Department of Gastroenterology, Norfolk and Norwich University Hospital NHS Trust, Norwich, United Kingdom

${ }^{7}$ Department of Medical Epidemiology and Biostatistics, Karolinska Institutet, Stockholm, Sweden

${ }^{8}$ Department of Pediatrics, Örebro University Hospital, Örebro University, Örebro, Sweden ${ }^{9}$ Pediatric Gastroenterology and Nutrition Unit, Sachs' Children's Hospital, Stockholm, Sweden 10 Department of Surgical Sciences, Uppsala University, Uppsala, Sweden

\section{Corresponding Author:}

Hamed Khalili, MD MPH

Massachusetts General Hospital, Gastroenterology Unit

Crohn's and Colitis Center

165 Cambridge Street, $9^{\text {th }}$ Floor

Boston, MA 02114

Phone: 617-726-4951; Fax: 978-882-6710

Email Address: hkhalili@mgh.harvard.edu

Word Count $=3751$

\section{Financial Support:}

This work was supported by the National Institute of Diabetes and Digestive and Kidney Diseases K24 DK098311 and a Crohn's and Colitis Foundation Senior Research Award to ATC. Dr. Chan is also the Stuart and Suzanne Steele MGH Research Scholar. We acknowledge the Swedish Infrastructure for Medical Population-based Life-course Environmental Research (SIMPLER) for provisioning of facilities and database. SIMPLER receives funding through the Swedish Research Council under the grant no 2017-00644.

\section{Potential Competing Interests:}


Hamed Khalili receives consulting fees from Abbvie. Hamed Khalili also receives grant support from Takeda and Pfizer. Andrew T. Chan receives consulting fees from Janssen, Pfizer Inc., and Bayer Pharma AG. Simon S. Chan has received consulting fees from Abbvie, Takeda and Ferring.

Ola Olén has been PI on projects at Karolinska Institutet partly financed by investigator-initiated grants from Janssen, Ferring, Takeda, and Pfizer. None of those studies have any relation to the present study. Karolinska Institutet has received fees for Olén's lectures and participation on advisory boards from Janssen, Ferring, Takeda, and Pfizer regarding topics not related to the present study. Ola Olén was supported by grants from the Swedish Medical Society (Project grants; Fund for Research in Gastroenterology; and Ihre Foundation), Mag-tarmfonden, Karolinska Institutet Foundations, Swedish Foundation for Strategic Research, and Regional Agreement on Medical Training and Clinical Research between Stockholm County Council and Karolinska Institutet (ALF). Jonas F. Ludvigsson coordinates a study on behalf of the Swedish IBD quality register (SWIBREG). This study has received funding from Janssen corporation. The remaining authors have no conflicts to disclose.

\section{Author Contributions:}

Hamed Khalili - Study concept and design, analysis and interpretation of data, drafting manuscript, critically revising manuscript

Niclas Hakansson - Data acquisition, critically revising manuscript

Simon S. Chan - Study concept and design, critically revising manuscript Ye Chen - Statistical analysis

Paul Lochhead - Study concept and design, critically revising manuscript Jonas F. Ludvigsson - Study concept and design, critically revising manuscript

Andrew T. Chan - Study concept and design, critically revising manuscript Ola Olen - Study concept and design, critically revising manuscript Andrew R. Hart - Study concept and design, critically revising manuscript Alicja Wolk - Study concept and design, data acquisition, analysis and interpretation of data, drafting manuscript, critically revising manuscript

\section{List of Abbreviations:}

Body mass index (BMI)

Cohort of Swedish Men (CoSM)

Crohn's disease (CD)

Crohn's disease exclusion diet (CDED)

European Prospective Cohort Study into Cancer and Diet (EPIC)

Exclusive enteral nutrition (EEN)

Hazard ratio (HR)

Inflammatory bowel disease (IBD)

Modified Mediterranean diet (mMED)

Nurses' Health Study

Semiquantitative food frequency questionnaire (SFFQ)

Specific Carbohydrate and Mediterranean Diets to Induce Remission of Crohn's Disease (DINECD)

Swedish Mammography Cohort (SMC)

Ulcerative colitis (UC) 


\section{ABSTRACT}

Objective: To examine the relationship between Mediterranean diet and risk of later-onset Crohn's disease (CD) or ulcerative colitis (UC).

Design: We conducted a prospective cohort study of 83,147 participants (age range: 45-79 years) enrolled in the Cohort of Swedish Men (CoSM) and Swedish Mammography Cohort (SMC). A validated food frequency questionnaire was used to calculate an adherence score to a modified Mediterranean diet (mMED) at baseline in 1997. Incident diagnoses of CD and UC were ascertained from the Swedish Patient Register. We used Cox proportional hazards modeling to calculate hazard ratios (HRs) and $95 \% \mathrm{Cl}$.

Results: Through December of 2017, we confirmed 164 incident cases of CD and 395 incident cases of UC with an average follow up of 17 years. Higher mMED score was associated with a lower risk of CD $\left(P_{\text {trend }}=0.03\right)$ but not UC $\left(P_{\text {trend }}=0.61\right)$. Compared to participants in the lowest category of mMED score (0-2), there was a statistically significant lower risk of CD (HR $=0.42$, $95 \% \mathrm{Cl} 0.22-0.80)$ but not UC ( $\mathrm{HR}=1.08,95 \% \mathrm{Cl} 0.74-1.58)$. These associations were not modified by age, sex, education level, body mass index, or smoking (All $\mathrm{P}_{\text {interaction }}>0.30$ ). The prevalence of poor adherence to a Mediterranean diet (mMED score $=0-2$ ) was $27 \%$ in our cohorts, conferring a population attributable risk of $12 \%$ for later-onset CD.

Conclusion: In two prospective studies, greater adherence to a Mediterranean diet was associated with a significantly lower risk of later-onset CD. 
What is already known about the topic:

Diet is widely thought to play an important role in the pathogenesis of inflammatory bowel disease.

Several epidemiologic studies have identified fiber intake to be protective against Crohn's disease.

What are the new findings:

Greater adherence to a Mediterranean diet is associated with a lower risk of later onset Crohn's disease.

In two large prospective cohorts in Sweden, poor adherence to a Mediterranean diet conferred a population attributable risk of $12 \%$ for later-onset Crohn's disease.

How might it impact on clinical practice in the foreseeable future?

Our findings further highlight the importance of continued research focusing on the benefits of the Mediterranean diet among individuals at risk of developing Crohn's disease. 


\section{INTRODUCTION:}

The pathogenesis of Crohn's disease (CD) and ulcerative colitis (UC), collectively known as inflammatory bowel disease (IBD), is thought to be related to an inappropriate immune response to the gut microbiota in a genetically susceptible host. Diet, through its interaction with the gut microbiome and host barrier function and immunity, plays an important role in the pathogenesis of $\mathrm{IBD}^{1}$. There are also accumulating data on the effectiveness of dietary strategies such as exclusive enteral nutrition (EEN) and the CD Exclusion Diet (CDED) in treatment of active $C^{1-4}$, further highlighting the critical role of diet in IBD. Nevertheless, prior epidemiologic studies of the relationship between diet and IBD have yielded very few plausible causal relationships (reviewed in $\left.{ }^{5}\right)$. The majority of these studies have focused on individual food groups and nutrients. In contrast, studies that focus on characterizing the link between dietary patterns and risk of IBD can take into account overall eating patterns which represent the totality of all foods and beverages consumed, therefore preserving the complexities and potential for synergism between dietary components.

Recently, the Crohn's and Colitis Foundation has undertaken an initiative to study the therapeutic effectiveness of Mediterranean diet in patients with established IBD. However, the relationship between adherence to a Mediterranean diet, characterized by high intakes of fruit and vegetables, whole grains, polyunsaturated fat, and proteins from fish, legumes, and nuts, and incident IBD has not been widely explored. Several lines of evidence suggest a role for Mediterranean diet in both prevention and treatment of IBD. First, ecologic studies have demonstrated lower incidence of IBD in Southern Europe where there is a higher consumption of a Mediterranean diet (reviewed in ${ }^{6}$ ). Second, previous studies have shown that a 
Mediterranean diet reduces plasma levels of inflammatory markers, such as high sensitivity Creactive protein and tumor necrosis factor alpha and the risk of development and progression of other immune-mediated disorders including psoriasis and rheumatoid arthritis ${ }^{7-10}$. Since IBD is characterized by a subclinical disease state marked by elevation in inflammatory and serological markers ${ }^{11}, 12$, adherence to a Mediterranean diet may have a plausible benefit in preventing development of clinically significant disease. Third, at least one small pilot study $(n=8)$ has demonstrated improvement in inflammatory markers, gene expression, and the gut microbiome in $\mathrm{CD}$ patients who consume a Mediterranean diet for 6 weeks ${ }^{13}$. Lastly, CDED has been shown to have therapeutic benefits in patients with $C D^{1}$, and is comprised of components (i.e. high intake of fruit and vegetables and low intake of red and processed meat) that overlap with Mediterranean diet.

We therefore sought to investigate the association between relative adherence to a Mediterranean diet and risk of incident $C D$ and $U C$ in two large prospective cohorts of men and women in Sweden. With detailed and validated data on dietary information on over 80,000 middle-aged men and women, these cohorts offered us a unique opportunity to examine the relationship between diet and later-onset IBD, where relative to younger-onset disease the overall contribution of environment is significantly greater ${ }^{14,15}$. 


\section{METHODS:}

\section{Study population:}

Swedish Mammography Cohort (SMC) is a population-based prospective cohort study established between 1987 and 1990 in the Uppsala county of central Sweden. Briefly, all women who lived in Uppsala County and were born from 1914 and 1948 received an invitation by mail to participate in a mammography screening program. A total of 66,651 women $(74 \%$ response rate), age 40-74 years, returned completed questionnaires on diet, alcohol, weight, height, and reproductive and menopausal factors. Additional data on other lifestyle factors (e.g. smoking, physical activity, etc.), medications, and medical illnesses were collected in 1997, 2008, and 2009. Cohort of Swedish Men (CoSM) is a parallel prospective cohort of 45,906 men, age 45-79 years, established in Örebro and Västmanland Counties in Central Sweden in the autumn of 1997. Similar to SMC, all men born between 1918 and 1952 in these counties received an invitation to participate in this study. Similar to SMC, participants provided information on diet and lifestyle factors (e.g. physical activity, smoking, etc) at baseline in 1997. Follow up questionnaires were administered in 2008 and 2009. Our study population included participants who had completed the 1997 questionnaires in both cohorts, which included all participants in CoSM and women in SMC who were still alive in 1997 and returned the dietary questionnaire $(n=38,984,70 \%$ response rate). We excluded participants with a diagnosis of IBD prior to baseline, who did not provide information on diet or had implausible total caloric intake defined by intakes not within three standard diviations of the log transformed mean (Figure 1).

\section{Primary Exposure and Other Covariates:}


In both cohorts, dietary data were collected using a 96-item semiquantitative food frequency questionnaire (SFFQ) at baseline in 1997 (validation studies described below) as previously described ${ }^{16}$. Participants reported average frequency of consumption of each food item in the previous year. There were 8 categories for frequency of intake ranging from zero servings per month to up to three times per day. Serving sizes for frequently consumed food groups (e.g. bread, coffee, tea, etc.) were prespecified. For other food groups, serving frequency was converted to average daily intake based on age- and sex-specific portion sizes and reference data from the Swedish National Food Agency database ${ }^{17}$.

We calculated a modified Mediterranean diet score (mMED score), ranging from 0 to 8 , based on relative adherence to a traditional Mediterranean diet adapted from a diet scale originally reported by Trichopoulou and colleagues ${ }^{18,19}$. One point was given for intakes above the median for (1) fruit and vegetables (apple, banana, berry, orange/citrus, and other fruit; carrot, beetroot, broccoli, cabbage, cauliflower, lettuce, onion, garlic, pepper, spinach, tomato, and other vegetables); (2) legumes (beans, pea soup, and lentils) and nuts; (3) non-refined or high-fiber grains (whole meal bread, crisp bread, oatmeal, and wheat bran); (4) fermented dairy products (cultured milk, yogurt, and cheese); and (5) fish. Additionally, one point was given for (6) intakes below the median of red and processed meat, for (7) use of olive or rapeseed oil for cooking or as dressing, and for (8) moderate alcohol consumption with an average of 5 to 15 grams of alcohol per day.

The SFFQ has been validated against fourteen 24-hr diet recall interviews conducted over a one-year period in a subset of participants in $\operatorname{CoSM}(n=248)$. The Spearman coefficient correlations between SFFQ and fourteen 24-hr recall interview ranged between 0.70 to 0.81 for 
intakes of total carbohydrate, fat, fiber, and alcohol ${ }^{20}$. Additionally, the reproducibility of two SFFQ completed one year apart was good with intraclass correlation ranging from 0.65 to 0.85 for all macronutrients. In SMC, the SFFQ (version from 1987 similar to that used in CoSM and SMC in 1997) has been validated against four one-week diet records done over a one-year period in 129 women $^{21}$. The Spearman correlation coefficient between SFFQ and diet records for ranged from 0.63 to 0.73 comparing dietary patterns (i.e. prudent versus western) and 0.40 to 0.82 for main food groups in a Mediterranean diet. Additionally, the reproducibility of these patterns between two SFFQ completed one year apart ranged from 0.41 to 0.73 , consistent with other studies in Asia and the US ${ }^{22-24}$. Validated data on weight, height, and physical activity and detailed information on smoking and education level were also collected at baseline in both cohorts as previously described ${ }^{25-30}$. Lastly, information on date of death and emigration was collected from the Swedish Total Population Register ${ }^{31}$.

\section{Outcome Ascertainment:}

The Swedish Patient Register has collected nationwide data on hospital discharges since $1987^{32}$. Each entry represents an encounter and includes date of birth, sex, dates of hospital admission, hospital department, and discharge diagnoses (including surgical procedures) ${ }^{32}$. All encounters are organized according to individual's personal identify number. Starting in January of 2001, the Swedish Patient Register also included data on all non-primary care outpatient diagnoses and procedures ${ }^{33}$.

Incident cases of $C D$ and $U C$ were ascertained through linkage of SMC and CoSM participants to the Swedish Patient Register and were defined by at least two inpatient or outpatient encounters with a primary or secondary diagnosis (for UC ICD9: '556' or ICD10: 'K51'; 
for CD ICD9: 555 or ICD10: K50) following the return of baseline questionnaires in 1997 in both cohorts. Use of ICD coding for identifying a number of chronic diseases, including IBD, in the inpatient component of the Swedish Patient Register has a positive predictive value of $85-95 \%{ }^{34}$. Additionally, in a recent validation study designed specifically for IBD, using both inpatient and outpatient components of the Swedish Patient Register, the positive predictive values using our definition for CD, UC, and IBD cases were $81 \%, 90 \%$, and $93 \%$ respectively ${ }^{35}$.

\section{Statistical Analysis:}

Follow up time was defined from January 1, 1998 to date of diagnosis, emigration, death, or end of follow up (December 31, 2017), whichever came first. We assessed the mMED score as a quantitative exposure and categorized as $0-2,3-4,5$, and $6-8$. These categories were selected $a$ priori to reflect extreme scores and to ensure an adequate number of participants in each group based on prior studies of mMED score in these cohorts ${ }^{36,37}$. The cutoffs for the lower and upper categories also correspond to the lower and upper quartiles of the mMED score distribution. Additionally, we examined for non-linear associations between mMED score and risk of CD and UC using restricted cubic splines ${ }^{38}$ and observed no evidence for such associations. We also used multiple imputations with chain equations to carry out 20 imputations of missing data on covariates. The proportion of missing data in our study was 9495 (11\%) for physical activity, 2969 (3.5\%) for BMI, 1306 (1.5\%) for smoking, and $182(0.2 \%)$ for education level.

We used Cox proportional hazards modeling to estimate the age- and multivariable (MV)adjusted hazard ratio (HR) and 95\% confidence interval (CI). Our models were adjusted for age, body mass index (BMI), education level, smoking, total caloric intake, and physical activity (Methr/week). Additionally, all models were stratified by sex (i.e. cohort). Test for trend across 
categories was conducted by assigning the median value to each mMED category and modeling this as a continuous variable. We performed several sensitivity analyses. We restricted our follow up to after January 2002 to account for the introduction of outpatient encounters in the Swedish Patient Register and to allow for one year gap for identifying prevalent cases of IBD previously not captured through inpatient register. In addition, this analysis allowed us to assess for the possibility of reverse causation related to participants' dietary changes as a result of symptoms related to undiagnosed subclinical or early disease. We also examined the possibility that the association between Mediterranean diet and risk of IBD may differ according to subgroups defined by age, sex, BMI, and smoking. We tested for the significance of the interaction by entering mMED score and these covariates in our models as multiplicative interaction terms. Finally, we calculated the population attributable risk conferred by a relatively poor adherence to the Mediterranean diet (i.e. mMED score $=0-2$ ), to estimate the percentage of IBD cases that might have been prevented if all participants had followed a Mediterranean diet, assuming a causal relationship ${ }^{39,40}$. We used SAS version 9.4 (Cary, NC) for these analyses. P-values were 2sided and values less than 0.05 were considered statistically significant. The study was approved by the regional ethics committee of Stockholm, Sweden. In both cohorts, consent to participate in the study was obtained through returned questionnaires. 


\section{RESULTS:}

After exclusions, 83,147 participants were eligible for our analyses. Through December of 2017, we confirmed 164 incident cases of CD and 395 incident cases of UC, yielding an incidence rate of 12 cases/100,000 person-years and 28 cases/100,000 person-years for CD and UC, respectively. The mean follow up time of participants was 17 years ( $s t d=5)$. The age at diagnosis of $C D$ and UC ranged from 47 to 83 years. Baseline characteristics of participants are reported in Table 1. Compared to participants in the lowest category of mMED score, those in the highest category on average had a higher caloric intake and education level and were more likely to be female and have never smoked. There were no significant differences in age, BMI, or physical activity.

In our age-adjusted model, we found that a higher mMED score was associated with a lower risk of $C D\left(P_{\text {trend }}=0.02\right)$ (Table 2). Specifically, compared to participants in the lowest category of mMED score, the age-adjusted HRs of CD were 0.70 (95\% Cl $0.49-0.99), 0.78(95 \% \mathrm{Cl}$ 0.50-1.23), and $0.42(95 \% \mathrm{Cl} 0.22-0.78)$ for participants with a mMED score of $3-4$, 5, and 6-8, respectively. These associations were not altered after adjusting for potential confounders including education level, BMI, total caloric intake, smoking, physical activity, and cohort (sex) $\left(P_{\text {trend }}=0.03\right)$. The MV-adjusted HRs were $0.69(95 \% \mathrm{Cl} 0.48-0.99), 0.78(95 \% \mathrm{Cl} 0.49-1.24)$, and $0.42(95 \% \mathrm{Cl} 0.22-0.80)$ for participants with a mMED score of 3-4, 5, and 6-8, respectively. We estimated that for every one-unit increase in mMED score, the MV-adjusted HR of CD was 0.87 (95\% Cl 0.78-0.96). Overall, the prevalence of relatively poor adherence to a Mediterranean diet (i.e. $\mathrm{mMED}$. Score $=0-2$ ) was $27 \%$ at baseline, conferring an adjusted population attributable risk of $12 \%$ (95\% Cl 3-26\%) for later-onset CD, assuming a causal relationship between diet and CD. 
In contrast, we did not observe an association between mMED score and risk of UC $\left(P_{\text {trend }}=0.61\right)$.

Compared to participants in the lowest category of mMED score, the MV-adjusted HRs of UC were 1.35 (95\% Cl 1.04-1.76), 1.37 (95\% Cl 0.99-1.90), and 1.08 (95\% Cl 0.74-1.58) for participants with a mMED score of 3-4, 5, and 6-8, respectively.

In exploratory analyses, we examined the association between individual components of mMED score and risk of CD and UC (Figure 2). Compared to participants with below the median consumption of nuts and legumes, the MV-adjusted HR of CD among participants with above the median consumption was $0.70,(95 \% \mathrm{Cl} 0.50-0.98)$. Higher consumption (above the median) of fruit and vegetable (MV-adjusted $H R=0.83,95 \% \mathrm{Cl} 0.60-1.15)$, fermented dairy (MV-adjusted HR $=0.82,95 \% \mathrm{Cl}-0.59-1.13)$, and non-refined grains ( $\mathrm{MV}$-adjusted $\mathrm{HR}=0.78,95 \% \mathrm{Cl} 0.55-1.10)$ and use of olive oil (MV-adjusted $\mathrm{HR}=0.70,95 \% \mathrm{Cl} 0.50-0.98$ ) were also associated with decreased risk of $C D$, albeit most estimates did not reach statistical significance. In contrast, we did not observe an association between any of the components of mMED score and risk of UC. We explored whether the association between mMED score and risk of CD and UC were consistent across several subgroups defined by age, sex (cohort), smoking, physical activity, or BMI (Tables 3 and 4) and observed no evidence for effect modification (All Pinteraction $>0.50$ ).

We conducted several sensitivity analyses. First, restricting our follow up to after January 1, 2002 to account for dietary changes that might have resulted from subclinical or undiagnosed disease (i.e. excluding cases of $C D$ and UC that were diagnosed within 4 years of SFFQ administration) did not materially alter our effect estimates. Specifically, compared to participants in the lowest category of mMED score, the MV-adjusted HRs of CD and UC in the highest category of mMED score were 0.44 (95\% Cl 0.23-0.84) and 1.14 (95\% Cl 0.77-1.69), 
respectively. Second, because of the potential differential effect of fruit and vegetables on risk of IBD, we recalculated mMED score by separating these two food groups. Compared to participants in the lowest category of mMED score (0-2), the MV-adjusted HRs in the highest group of mMED group (7-9) were $0.57(95 \% \mathrm{Cl} 0.32-0.99)$ for $\mathrm{CD}$ and 1.01 (95\% Cl $0.70-1.46)$ for UC. Third, as rapeseed oil was not included in the original definition of adherence to a Mediterranean diet, we conducted a sensitivity analysis excluding this from our calculation of mMED score. Compared to participants in the lowest category of mMED score (0-2), the MVadjusted HRs in the highest group of mMED group (6-8) were 0.44 (95\% $\mathrm{Cl} 0.23-0.85)$ for CD and 1.07 (95\% Cl $0.73-1.57)$ for UC. Finally, using a previously described array-based approach, we estimated that an unmeasured confounder would need to have a prevalence of $80 \%$ in the highest quartile of mMED score compared to $20 \%$ in the lowest quartile with a relative risk for $C D$ of less than 0.17 to account for our observed association ${ }^{41}$. 


\section{DISCUSSION:}

In two large prospective cohorts of middle-aged men and women in Sweden, we demonstrate that greater adherence to a Mediterranean diet is inversely associated with risk of later-onset CD but not UC. These findings were consistent across multiple sensitivity and subgroup analyses.

To our knowledge, this is the first prospective cohort study that has examined the relationship between a Mediterranean diet and risk of incident CD and UC. Nevertheless, one prior study from the EPIC investigators did not show an association between a Mediterranean dietary pattern and risk of $C D^{42}$. However, smaller numbers of $C D$ cases and significant heterogeneity in methods used to collect dietary information across centers in EPIC-IBD's nested case-control design may have limited the power of the study to find a significant association. Additionally, the influence of a Mediterranean diet on risk of CD may not be as pronounced in populations that are largely adherent to such a diet. In contrast, several prior studies have shown a relationship between individual food groups and nutrients, found in high quantity in a Mediterranean diet and risk of $\mathrm{IBD}^{43}, 44$. One prior study from the US prospective cohort studies of the Nurses' Health Study (NHS) and NHSII found that higher intake of fiber particularly from cruciferous vegetables and cereals is associated with a decreased risk of incident $\mathrm{CD}^{43}$. Similarly, data from the European Prospective Cohort Study into Cancer and Diet (EPIC) have shown an inverse association between fiber derived from cereals and risk of CD among non-smokers ${ }^{44}$.

Our findings have plausible biologic mechanisms. Several dietary intervention studies have demonstrated significant changes in inflammatory markers, immune cell populations, and 
response to oxidative stress with Mediterranean diet ${ }^{8,45}$. Specifically, in Healthy Lifestyle in Europe by Nutrition in Adolescence (HELENA) study, adherence to a Mediterranean diet was associated with an attenuation in oxidative stress response in adolescents ${ }^{45}$. Similarly, in the randomized controlled trial of European Project on Nutrition in Elderly People (NU-AGE), adherence to a Mediterranean diet for one year was associated with significant changes in innate and adaptive immunity as measured by T cell degranulation, cytokine production, and co-receptor expression ${ }^{8}$. These results are further supported by a number of cross sectional analyses of patients with immune-mediated disorders including $C D$ and psoriasis demonstrating an inverse relationship between adherence to a Mediterranean diet and disease activity ${ }^{9,46}$. Additionally, adherence to a Mediterranean diet appears to have significant beneficial effect on the gut microbiome composition and function ${ }^{47,48}$. Lastly, components of a Mediterranean diet such as fiber may also exert anti-inflammatory effect through modification of the barrier function and bacterial translocation (reviewed in ${ }^{49}$ ).

Our observation that the beneficial association of the Mediterranean diet appears to be exclusive to $C D$ is in line with prior studies demonstrating that diet appears to be a stronger modifiable lifestyle factor for $\mathrm{CD}^{50}$. Additionally, dietary intervention studies such as $\mathrm{EEN}$ and CDED have primarily been effective in $\mathrm{CD}^{1-4}$. Although the exact biologic rationale behind this unique relationship is unclear, it may be explained in part by the greater role of the gut microbiome in $C D$; specifically, $C D$ is characterized by significant dysbiosis and further by improvement in disease activity with fecal diversion, as compared to UC $\mathrm{C}^{51-53}$. Therefore, dietinduced changes in the gut microbiome may have a greater impact in preventing and treating CD. 
We highlight several strengths of our study. First, the prospective nature minimized the risk of selection and recall biases that are commonly observed in cross-sectional studies of diet in IBD. Second, in our study we were able to account for other important lifestyle factors such as BMI, physical activity, and smoking that are likely to confound the relationship between diet and IBD. Lastly, we used a nationwide registry and a validated method to ascertain cases of CD and UC minimizing the risk of outcome misclassification.

We acknowledge several limitations. First, our study population included mostly middleaged men and women and therefore it is unclear whether our findings may be generalizable to younger individuals at risk of IBD. Nevertheless, environmental factors may play a greater role in development of later-onset $\mathrm{IBD}^{15}$, highlighting the importance of identifying modifiable risk factors in elderly-onset disease. Further, many of the beneficial anti-inflammatory, immunologic, and metabolic effects of Mediterranean diet have specifically been demonstrated in older adults $^{8}$. Second, calculation of mMED score was based on the distribution of dietary intake in our cohorts, which may not be generalizable to other populations with a vastly different pattern of dietary intake. As an example, comparing the median intake of the components of aMED score to those published by Trichopoulou and colleagues ${ }^{18}$ showed similar consumption of fruit and vegetable, red and processed meat, fish, and alcohol but vastly different intakes of legumes, nuts, and grains. Third, there are measurement errors associated with collection of dietary data. However, as demonstrated in our prior validation studies, when compared to other methods, SFFQ provides reasonably valid estimates of dietary intake. Additionally, measurement error for diet is unlikely to be systematically associated with the outcome and therefore commonly results in a spurious under-estimate of associations, rather than false over-estimates ${ }^{54}$. Hence, the 
inverse association with CD may be greater than we detected. Fourth, we do not have updated dietary data. However, as has been shown in other studies, individuals' dietary intake remains relatively stable over time ${ }^{55}$ and therefore it's less likely that their categorization based on diet will significantly change over time. Lastly, we acknowledge that our observed associations may be related to residual confounding related to our inability to adjust for other factors such as family history of IBD and early life exposures. However, adjusting for known confounders did not materially alter our estimates. Additionally, in our sensitivity analysis, we demonstrated that it will be very unlikely for an unmeasured confounder to fully attenuate the observed association between mMED score and risk of CD.

\section{Conclusion:}

In two large prospective cohort studies, we show that a greater adherence to a Mediterranean diet is associated with a lower risk of later onset CD. Our study further highlights the importance of continued research focusing on the benefits of the Mediterranean diet in patients with established CD. Such efforts including the ongoing clinical trial of Specific Carbohydrate and Mediterranean Diets to Induce Remission of Crohn's Disease (DINE-CD) will provide significant insight into the role of diet in IBD therapeutics.

Figure 1: Flow Chart of eligible participants in the study

*Abbreviations: Cohort of Swedish Men (COSM) and Swedish Mammography Cohort (SMC)

Figure 2: The Association Between Adherence to Individual Components of mMED Score and Risk of CD and UC*

*For fruit and vegetables, legumes \& nuts, non-refined/high fiber grains, fermented dairy, and fish the estimates represent comparison of above the median consumption, representing a score $=1$ for $\mathrm{mMED}$ score to below the median consumption. For red \& processed meat, the estimate represents comparison of below the median consumption, representing a score $=1$ for mMED score to above the median consumption. Moderate alcohol consumption is defined as 5-15 g of consumption per day. 


\begin{tabular}{|c|c|c|c|c|}
\hline & & mMED & & \\
\hline & $\begin{array}{c}(0-2) \\
(n=22,237)\end{array}$ & $\begin{array}{c}(3-4) \\
(n=36,951)\end{array}$ & $\begin{array}{c}(5) \\
(n=13,630)\end{array}$ & $\begin{array}{c}(6-8) \\
(n=10,329)\end{array}$ \\
\hline Age (yrs) & $62(10)$ & $61(10)$ & $61(9)$ & $60(9)$ \\
\hline Sex (female), \% & 36 & 45 & 53 & 61 \\
\hline \multicolumn{5}{|l|}{ Education, \% } \\
\hline Primary school & 80 & 73 & 65 & 57 \\
\hline High school & 10 & 11 & 12 & 13 \\
\hline University & 10 & 16 & 23 & 30 \\
\hline Body Mass Index $\left(\mathrm{kg} / \mathrm{m}^{2}\right)$ & $26(4)$ & $26(4)$ & $25(3)$ & $25(3)$ \\
\hline Ever smoking, \% & 60 & 55 & 52 & 49 \\
\hline Physical activity (Met-hr/wk) & $42(5)$ & $42(5)$ & $42(5)$ & $42(5)$ \\
\hline Total caloric intake & $2038(795)$ & $2256(848)$ & 2367 (847) & $2411(783)$ \\
\hline \multicolumn{5}{|l|}{ Components of mMED score } \\
\hline Fruit and vegetables (g/day) & $251(156)$ & $386(223)$ & $492(231)$ & $557(225)$ \\
\hline Legumes \& nuts (g/day), & $0.3(1)$ & $0.8(2)$ & $1(3)$ & $2(4)$ \\
\hline Non-refined/high fiber grains (g/day) & $137(115)$ & $190(131)$ & $210(128)$ & $221(121)$ \\
\hline Fermented dairy (g/day) & $151(182)$ & $253(240)$ & $325(260)$ & $376(256)$ \\
\hline Fish (g/day) & $26(29)$ & $37(37)$ & $43(33)$ & $48(30)$ \\
\hline Red \& processed meat (g/day) & $83(55)$ & $78(56)$ & $73(51)$ & $62(43)$ \\
\hline Olive oil and/or rapeseed oil use, $\%$ & 10 & 35 & 63 & 85 \\
\hline Moderate alcohol intake, $\%$ & 14 & 29 & 42 & 61 \\
\hline
\end{tabular}

*Abbreviations: years (yrs), standard deviation (std), Met-hours/week (Met-hr/wk), modified Mediterranean diet score (mMED score). All characteristics were derived from the 1997 questionnaires. Unless notes, continuous variables are presented in mean (standard deviation). 


\begin{tabular}{|c|c|c|c|c|c|}
\hline & \multicolumn{4}{|c|}{ mMED score } & \\
\hline & $\begin{array}{c}(0-2) \\
(n=22,237)\end{array}$ & $\begin{array}{c}(3-4) \\
(n=36,951)\end{array}$ & $\begin{array}{c}(5) \\
(n=13,630)\end{array}$ & $\begin{array}{c}(6-8) \\
(n=10,329)\end{array}$ & \\
\hline & & & & & $P_{\text {trend }}{ }^{9}$ \\
\hline Person-years of follow up & 361,082 & 624,597 & 238,698 & 185,790 & \\
\hline \multicolumn{6}{|l|}{ Crohn's disease } \\
\hline Number of cases & 56 & 67 & 29 & 12 & \\
\hline Age-adjusted, HR (95\% Cl) & 1.0 & $0.70(0.49-0.99)$ & $0.78(0.50-1.23)$ & $0.42(0.22-0.78)$ & 0.02 \\
\hline MV-adjusted, HR $(95 \% \mathrm{Cl})^{\Delta}$ & 1.0 & $0.69(0.48-0.99)$ & $0.78(0.49-1.24)$ & $0.42(0.22-0.80)$ & 0.03 \\
\hline \multicolumn{6}{|l|}{ Ulcerative colitis } \\
\hline Number of cases & 84 & 193 & 74 & 44 & \\
\hline Age-adjusted, HR (95\% CI) & 1.0 & $1.34(1.03-1.73)$ & $1.33(0.98-1.82)$ & $1.02(0.71-1.46)$ & 0.81 \\
\hline MV-adjusted, HR $(95 \% \mathrm{Cl})^{\Delta}$ & 1.0 & $1.35(1.04-1.76)$ & $1.37(0.99-1.90)$ & $1.08(0.74-1.58)$ & 0.61 \\
\hline
\end{tabular}

*Abbreviations: Multivariable (MV), hazard ratio (HR), confidence intervals (Cl). $\Delta$ Models are adjusted for cohort (sex), age (years), education (primary school, high school, and education), body mass index, smoking (never, past, and current), and total caloric intake. ๆ Ptrend was calculated using the median value for each category. 


\section{Table 3: mMED Score and Risk of Crohn's Disease According to Selected Strata*}

$\begin{array}{llll}\text { Cases } & \text { Person-years } & \mathrm{MV} \text {-adjusted } \mathrm{HR}, 95 \% \mathrm{Cl}^{\Delta} & \mathrm{P}_{\text {interaction }}\end{array}$

Sex

- Male

- Female

Age at Start of Follow up

- $<60$ years

Body mass index

- $\quad<25 \mathrm{~kg} / \mathrm{m}^{2}$

- $\quad \geq 25 \mathrm{~kg} / \mathrm{m}^{2}$

Education

- Primary school

- High school

- University
- $\geq 60$ years
$99 \quad 749,531$

65

101

63

79

85

120

20

24

77

56

60

101
660,637

773,622

636,546

677,777

732,391

979,353

162,981

267,834

547,956

558,895

623,266

765,627
$0.32(0.11-0.89)$

0.50 (0.21-1.21)

$0.45(0.21-0.97)$

0.31 (0.09-1.07)

$0.32(0.13-0.79)$

$0.56(0.22-1.38)$

0.52

$0.45(0.20-1.02)$

$0.49(0.09-2.57)$

0.46 (0.11-1.99)

0.99

$0.33(0.13-0.83)$

$0.61(0.21-1.78)$

$0.83(0.35-1.95)$

$0.17(0.05-0.57)$

0.78

0.84

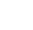

- $\quad$ Never smoke

*Abbreviations: Multivariable (MV), hazard ratio (HR), confidence intervals (Cl). $\Delta$ Comparing the extremes of mMED score quartiles. Models are adjusted for age (years), cohort (sex), body mass index, smoking (never, past, and current), and total caloric intake. ๆ Missing data on these variables were not included or imputed for these analyses. 


\section{Table 4: mMED Score and Risk of Ulcerative Colitis According to Selected Strata*}

Cases Person-years

MV-adjusted $\mathrm{HR}, 95 \% \mathrm{Cl}^{\triangle}$

Pinteraction

Sex

- Male

- Female

Age at Start of Follow up

- $<60$ years

- $\quad \geq 60$ years

Body mass index"

- $<25 \mathrm{~kg} / \mathrm{m}^{2}$

- $\quad \geq 25 \mathrm{~kg} / \mathrm{m}^{2}$

Education

- Primary school

- High school

- University

Physical activity

- < below the median

- $\quad \geq$ below the median
252

143

247

148

173

222

278

42

75

168

157

104

288
749,531

660,637

773,622

636,546

677,777

732,391

979,353

162,981

267,834

547,956

558,895

623,266

765,627
$1.20(0.74-1.96)$

0.98 (0.52-1.84)

0.96 (0.59-1.57)

$1.32(0.73-2.41)$

0.85

0.97

$1.10(0.63-1.93)$

1.09 (0.65-1.84)

1.25 (0.79-1.99)

1.71 (0.37-7.89)

0.77 (0.35-1.71)

$1.30(0.76-2.21)$

0.99 (0.51-1.92)

0.52

$1.03(0.49-2.17)$

$1.17(0.75-1.83)$

0.57

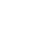

(1)

- Never smoker

- Ever smoker

\footnotetext{
*Abbreviations: Multivariable (MV), hazard ratio (HR), confidence intervals (CI). $\Delta$ Comparing the extremes of mMED score quartiles. Models are adjusted for age (years), cohort (sex), body mass index, smoking (never, past, and current), and total caloric intake. ๆ Missing data was not included or imputed for these analyses.
} 
Figure 1: Flow Chart of eligible participants in the study

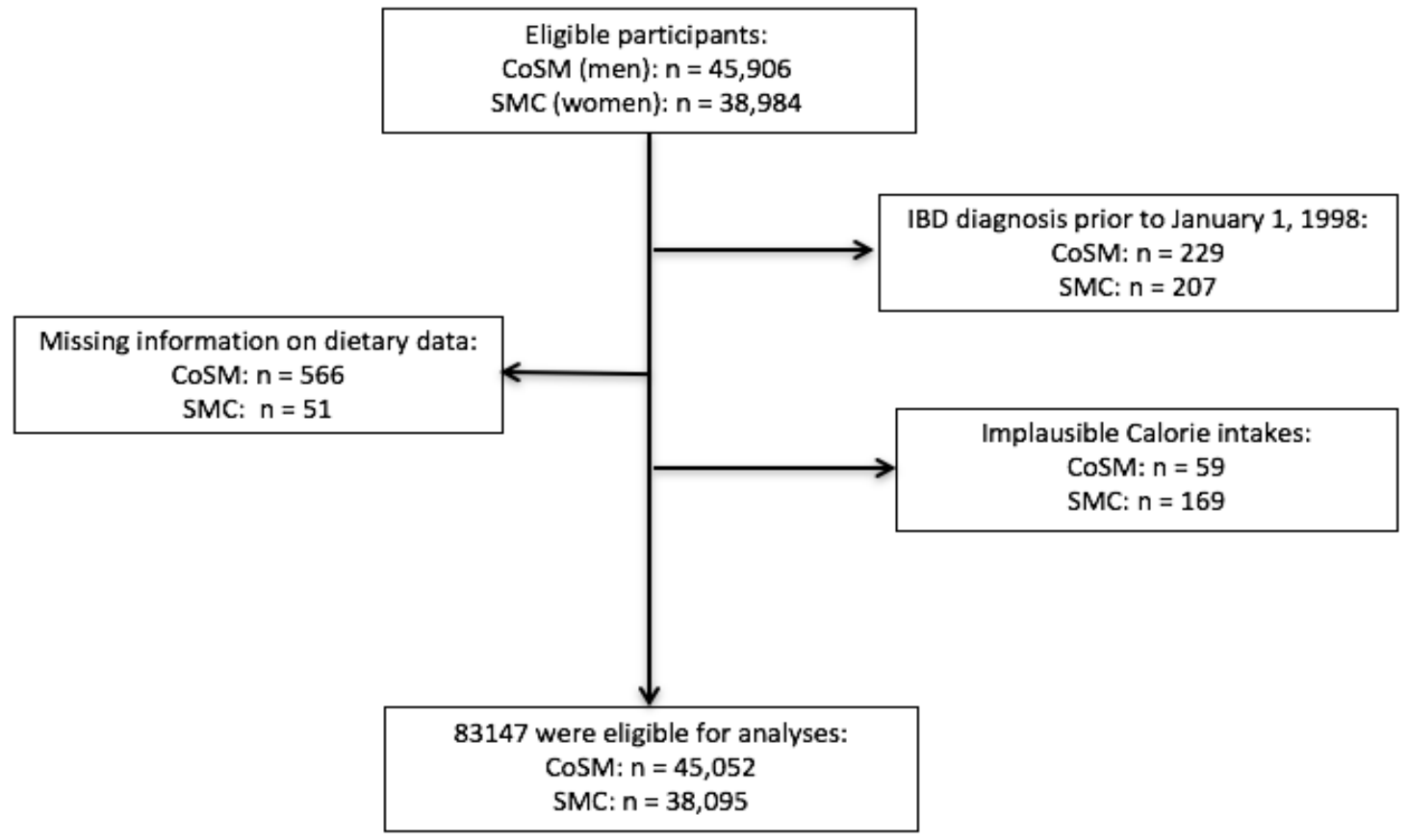

Figure 2. HRs for Crohn's disease and Ulcerative Colitis

\begin{tabular}{|l|c|c|}
\hline & \multicolumn{1}{|c|}{ Crohn's disease } \\
\hline $\begin{array}{l}\text { Fruit and vegetables } \\
\text { Legumes \& nuts } \\
\text { Non-refined/high fibre grains } \\
\text { Fermented dairy }\end{array}$ \\
$\begin{array}{l}\text { Fish } \\
\text { Red \& processed meat } \\
\text { Use of olive or rapeseed oil } \\
\text { Moderate alcohol intake }\end{array}$
\end{tabular}




\section{REFERENCES:}

1. Levine A, Wine E, Assa A, et al. Crohn's Disease Exclusion Diet Plus Partial Enteral Nutrition Induces Sustained Remission in a Randomized Controlled Trial. Gastroenterology 2019.

2. Svolos V, Hansen R, Nichols B, et al. Treatment of Active Crohn's Disease With an Ordinary Food-based Diet That Replicates Exclusive Enteral Nutrition. Gastroenterology 2019;156:1354-1367 e6.

3. Borrelli O, Cordischi L, Cirulli M, et al. Polymeric diet alone versus corticosteroids in the treatment of active pediatric Crohn's disease: a randomized controlled open-label trial. Clin Gastroenterol Hepatol 2006;4:744-53.

4. Takagi S, Utsunomiya K, Kuriyama S, et al. Effectiveness of an 'half elemental diet' as maintenance therapy for Crohn's disease: A randomized-controlled trial. Aliment Pharmacol Ther 2006;24:1333-40.

5. Khalili $\mathrm{H}$, Chan SSM, Lochhead $\mathrm{P}$, et al. The role of diet in the aetiopathogenesis of inflammatory bowel disease. Nat Rev Gastroenterol Hepatol 2018;15:525-535.

6. Burisch J, Jess $\mathrm{T}$, Martinato $\mathrm{M}$, et al. The burden of inflammatory bowel disease in Europe. J Crohns Colitis 2013;7:322-37.

7. Forsyth C, Kouvari M, D'Cunha NM, et al. The effects of the Mediterranean diet on rheumatoid arthritis prevention and treatment: a systematic review of human prospective studies. Rheumatol Int 2018;38:737-747.

8. Maijo M, Ivory K, Clements SJ, et al. One-Year Consumption of a Mediterranean-Like Dietary Pattern With Vitamin D3 Supplements Induced Small Scale but Extensive Changes of Immune Cell Phenotype, Co-receptor Expression and Innate Immune Responses in Healthy Elderly Subjects: Results From the United Kingdom Arm of the NUAGE Trial. Front Physiol 2018;9:997.

9. Phan C, Touvier M, Kesse-Guyot E, et al. Association Between Mediterranean Antiinflammatory Dietary Profile and Severity of Psoriasis: Results From the NutriNet-Sante Cohort. JAMA Dermatol 2018;154:1017-1024.

10. Sureda A, Bibiloni MDM, Julibert A, et al. Adherence to the Mediterranean Diet and Inflammatory Markers. Nutrients 2018;10.

11. Lochhead P, Khalili H, Ananthakrishnan AN, et al. Association Between Circulating Levels of C-Reactive Protein and Interleukin- 6 and Risk of Inflammatory Bowel Disease. Clin Gastroenterol Hepatol 2016;14:818-824 e6.

12. Choung RS, Princen F, Stockfisch TP, et al. Serologic microbial associated markers can predict Crohn's disease behaviour years before disease diagnosis. Aliment Pharmacol Ther 2016;43:1300-10.

13. Marlow G, Ellett S, Ferguson IR, et al. Transcriptomics to study the effect of a Mediterranean-inspired diet on inflammation in Crohn's disease patients. Hum Genomics 2013;7:24.

14. Li D, Haritunians T, Landers C, et al. Late-Onset Crohn's Disease Is A Subgroup Distinct in Genetic and Behavioral Risk Factors With UC-Like Characteristics. Inflamm Bowel Dis 2018;24:2413-2422. 
15. Ruel J, Ruane D, Mehandru S, et al. IBD across the age spectrum: is it the same disease? Nat Rev Gastroenterol Hepatol 2014;11:88-98.

16. Khalili H, Hakansson N, Chan SS, et al. No Association Between Consumption of Sweetened Beverages and Risk of Later-Onset Crohn's Disease or Ulcerative Colitis. Clin Gastroenterol Hepatol 2019;17:123-129.

17. Livsmedelsverket.

18. Trichopoulou A, Costacou T, Bamia C, et al. Adherence to a Mediterranean diet and survival in a Greek population. N Engl J Med 2003;348:2599-608.

19. Trichopoulou A, Kouris-Blazos A, Wahlqvist ML, et al. Diet and overall survival in elderly people. BMJ 1995;311:1457-60.

20. Messerer M, Johansson SE, Wolk A. The validity of questionnaire-based micronutrient intake estimates is increased by including dietary supplement use in Swedish men. J Nutr 2004;134:1800-5.

21. Khani BR, Ye W, Terry $P$, et al. Reproducibility and validity of major dietary patterns among Swedish women assessed with a food-frequency questionnaire. J Nutr 2004;134:1541-5.

22. Hu FB, Rimm E, Smith-Warner SA, et al. Reproducibility and validity of dietary patterns assessed with a food-frequency questionnaire. Am J Clin Nutr 1999;69:243-9.

23. Kusama K, Le DS, Hanh TT, et al. Reproducibility and validity of a food frequency questionnaire among Vietnamese in Ho Chi Minh City. J Am Coll Nutr 2005;24:466-73.

24. Nanri A, Shimazu T, Ishihara J, et al. Reproducibility and validity of dietary patterns assessed by a food frequency questionnaire used in the 5-year follow-up survey of the Japan Public Health Center-Based Prospective Study. J Epidemiol 2012;22:205-15.

25. Kuskowska-Wolk A, Karlsson $\mathrm{P}$, Stolt $\mathrm{M}$, et al. The predictive validity of body mass index based on self-reported weight and height. Int J Obes 1989;13:441-53.

26. Orsini N, Bellocco R, Bottai M, et al. Validity of self-reported total physical activity questionnaire among older women. Eur J Epidemiol 2008;23:661-7.

27. Di Giuseppe D, Bottai M, Askling J, et al. Physical activity and risk of rheumatoid arthritis in women: a population-based prospective study. Arthritis Res Ther 2015;17:40.

28. Byberg L, Melhus H, Gedeborg R, et al. Total mortality after changes in leisure time physical activity in 50 year old men: 35 year follow-up of population based cohort. BMJ 2009;338:b688.

29. Sadr-Azodi O, Orsini N, Andren-Sandberg A, et al. Abdominal and total adiposity and the risk of acute pancreatitis: a population-based prospective cohort study. Am J Gastroenterol 2013;108:133-9.

30. Sadr-Azodi O, Andren-Sandberg A, Orsini N, et al. Cigarette smoking, smoking cessation and acute pancreatitis: a prospective population-based study. Gut 2012;61:262-7.

31. Ludvigsson JF, Almqvist C, Bonamy AK, et al. Registers of the Swedish total population and their use in medical research. Eur J Epidemiol 2016;31:125-36.

32. Ludvigsson JF, Otterblad-Olausson P, Pettersson BU, et al. The Swedish personal identity number: possibilities and pitfalls in healthcare and medical research. Eur J Epidemiol 2009;24:659-67.

33. Socialstyrelsen. In English - the National Patient Register. 2011. 
34. Ludvigsson JF, Andersson E, Ekbom A, et al. External review and validation of the Swedish national inpatient register. BMC Public Health 2011;11:450.

35. Jakobsson GL, Sternegard E, Olen O, et al. Validating inflammatory bowel disease (IBD) in the Swedish National Patient Register and the Swedish Quality Register for IBD (SWIBREG). Scand J Gastroenterol 2017;52:216-221.

36. Byberg L, Bellavia A, Larsson SC, et al. Mediterranean Diet and Hip Fracture in Swedish Men and Women. J Bone Miner Res 2016;31:2098-2105.

37. Bellavia A, Tektonidis TG, Orsini N, et al. Quantifying the benefits of Mediterranean diet in terms of survival. Eur J Epidemiol 2016;31:527-30.

38. Harrell FE, Jr., Lee KL, Pollock BG. Regression models in clinical studies: determining relationships between predictors and response. J Natl Cancer Inst 1988;80:1198-202.

39. Spiegelman D, Hertzmark E, Wand HC. Point and interval estimates of partial population attributable risks in cohort studies: examples and software. Cancer Causes Control 2007;18:571-9.

40. Tamimi RM, Spiegelman D, Smith-Warner SA, et al. Population Attributable Risk of Modifiable and Nonmodifiable Breast Cancer Risk Factors in Postmenopausal Breast Cancer. Am J Epidemiol 2016;184:884-893.

41. Schneeweiss S, Glynn RJ, Tsai EH, et al. Adjusting for unmeasured confounders in pharmacoepidemiologic claims data using external information: the example of COX2 inhibitors and myocardial infarction. Epidemiology 2005;16:17-24.

42. Racine A, Carbonnel F, Chan SS, et al. Dietary Patterns and Risk of Inflammatory Bowel Disease in Europe: Results from the EPIC Study. Inflamm Bowel Dis 2016;22:345-54.

43. Ananthakrishnan AN, Khalili H, Konijeti GG, et al. A prospective study of long-term intake of dietary fiber and risk of Crohn's disease and ulcerative colitis. Gastroenterology 2013;145:970-7.

44. Andersen V, Chan S, Luben R, et al. Fibre intake and the development of inflammatory bowel disease: A European prospective multi-centre cohort study (EPIC-IBD). J Crohns Colitis 2018;12:129-136.

45. Arouca AB, Santaliestra-Pasias AM, Moreno LA, et al. Diet as a moderator in the association of sedentary behaviors with inflammatory biomarkers among adolescents in the HELENA study. Eur J Nutr 2018.

46. Papada E, Amerikanou C, Forbes A, et al. Adherence to Mediterranean diet in Crohn's disease. Eur J Nutr 2019.

47. De Filippis F, Pellegrini N, Vannini L, et al. High-level adherence to a Mediterranean diet beneficially impacts the gut microbiota and associated metabolome. Gut 2016;65:18121821.

48. Bowyer RCE, Jackson MA, Pallister $\mathrm{T}$, et al. Use of dietary indices to control for diet in human gut microbiota studies. Microbiome 2018;6:77.

49. Levine A, Sigall Boneh $\mathrm{R}$, Wine $\mathrm{E}$. Evolving role of diet in the pathogenesis and treatment of inflammatory bowel diseases. Gut 2018;67:1726-1738.

50. Lewis JD, Abreu MT. Diet as a Trigger or Therapy for Inflammatory Bowel Diseases. Gastroenterology 2017;152:398-414 e6.

51. Morgan XC, Tickle TL, Sokol H, et al. Dysfunction of the intestinal microbiome in inflammatory bowel disease and treatment. Genome Biol 2012;13:R79. 
52. Rutgeerts $P$, Goboes K, Peeters $M$, et al. Effect of faecal stream diversion on recurrence of Crohn's disease in the neoterminal ileum. Lancet 1991;338:771-4.

53. Lim AG, Langmead FL, Feakins RM, et al. Diversion colitis: a trigger for ulcerative colitis in the in-stream colon? Gut 1999;44:279-82.

54. Rosner B, Michels KB, Chen YH, et al. Measurement error correction for nutritional exposures with correlated measurement error: use of the method of triads in a longitudinal setting. Stat Med 2008;27:3466-89.

55. Wang DD, Leung CW, Li Y, et al. Trends in dietary quality among adults in the United States, 1999 through 2010. JAMA Intern Med 2014;174:1587-95. 